\title{
Lock-in Thermography on Electronic Devices Using Spatial Deconvolution
}

\author{
by M. Hejjo Al Rifai, O. Breitenstein and J.P. Rakotoniaina \\ Max Planck Institute of Microstructure Physics, Halle, Germany
}

\begin{abstract}
We show that the $" 0 \%-90^{\circ}$ " lock-in thermography signal is very useful to be displayed for microscopic investigation of integrated circuits (ICs). Like the phase signal this signal is independent on the local IR emissivity. But in contrast to it signal components from different heat sources are superpositioning linearly, which holds at least in good approximation. Therefore this signal can be deconvoluted (inverted) numerically for compensating the blurring caused by lateral heat diffusion.
\end{abstract}

\section{Introduction}

Microthermal analysis is a standard tool for failure analysis in electronic devices like integrated circuits (ICs) and solar cells. Many faults like gate breakthroughs, latch-ups, or shorts, are leading to characteristic local heat sources at the surface of these devices. The most popular techniques for such investigations are liquid crystal investigations [1] and fluorescent microthermal imaging [2], both representing steady-state methods. Also infrared (IR) thermography is sometimes used microscopically for IC investigations [3], but it has some disadvantages: Its spatial resolution is limited by the used IR wavelength range to the order of $5 \mu \mathrm{m}$, its sensitivity is limited to some $10 \mathrm{mK}$, and the images are strongly influenced by the IR emissivity contrast. There are special techniques available to compensate thermograms for the emissivity contrast [3], but these techniques are time-expensive, since they need thermostatting of the sample, and they are not always $100 \%$ efficient because of possible sample movement. Moreover, due to the inevitable lateral heat conduction in the silicon material, stationary thermograms always appear blurred. Hence the effective spatial resolution is usually worse than the pixel resolution.

Both the sensitivity and the effective spatial resolution can be dramatically improved if lock-in thermography is used instead of steady-state thermography [4]. These results can also be displayed as phase images, which are not disturbed by emissivity contrast anymore. Nevertheless, the blurring influence of the lateral heat conduction also remains here. Note that a measure of the travelling range of thermal 
waves is the thermal diffusion length, which in silicon for a typical lock-in frequency of $88 \mathrm{~Hz}$ is about $0.58 \mathrm{~mm}$ and reduces with $1 / \sqrt{ } \mathrm{f}_{\text {lock-in. }}$. Hence, at least for microscopic IR investigations lateral heat conductivity-induced image blurring represents a serious practical problem.

Mathematically, "blurring" can be described by a 2-dimensional convolution of a source image (here the 2-dimensional dissipated power distribution) with a radial point spread function. Thus, 2-dimensional spatial deconvolution (inversion) provides a possibility to "deblurr" images, if the cause of the blurring is known [5]. The conditions for applying deconvolution techniques are the following: 1 . The image formation mechanism has to be linear, hence signals of different heat sources have to superimpose linearly; 2 . the signal-to-noise ratio of the image has to be very good, since deconvolution always degrades the signal-to-noise ratio; and 3 . the image is not allowed to have artifacts like, e.g., emissivity contrast. In IR lock-in thermography condition No. 2 can usually be realized by choosing a sufficiently long acquisition time. However, since the amplitude image as well as the two primary images (inphase and out-of-phase image) are all modulated by the disturbing emissivity contrast, they do not match condition No. 3 and cannot be used for IC investigations. Also the phase image, which does not contain any emissivity contrast, cannot be used for deconvolution because it violates condition No. 1, since it is a highly nonlinear combination of the two primary signals, which does not behave linearly at all. Since the phase signal is a measure of the time delay between the pulsed excitation and the temperature modulation, for highly localized heat sources at the surface it is even independent on the magnitude of the heat source, as long as thermal waves of neighbouring heat sources do not significantly overlap.

\section{Evaluation of the " $0 \%-90^{\circ "}$ signal}

The wayout from this dilemma at least for microscopic investigations is to use a new kind of representation of lock-in thermography results, which is the in-phase image $\left(0^{\circ}\right.$ image) divided by the out-of-phase image $\left(-90^{\circ}\right.$ image). Note that in electronic device testing the $-90^{\circ}$ image is usually displayed as the out-of-phase signal instead of the $90^{\circ}$-image, since the latter one is essentially negative. The amplitudes of the real and the imaginary parts of a thermal wave around a point heat source at the surface of an infinitely thick body are given by [6]:

$$
0^{\circ}=\frac{\mathrm{A}}{\mathrm{r}} \exp \left(\frac{-\mathrm{r}}{\Lambda}\right) \cos \left(\frac{-\mathrm{r}}{\Lambda}\right)
$$


$-90^{\circ}=\frac{\mathrm{A}}{\mathrm{r}} \exp \left(\frac{-\mathrm{r}}{\Lambda}\right) \sin \left(\frac{-\mathrm{r}}{\Lambda}\right)$

with $\Lambda$ being the thermal diffusion length

$$
\Lambda=\sqrt{\frac{\lambda}{\rho c_{p} \pi f_{\text {lock-in }}}}
$$

Here $A$ is an amplitude factor being dependent on the power of the heat source, $r$ is the radial distance to the source, $\lambda$ ist the thermal conductivity of the material, $\rho$ is its density, and $c_{p}$ is its specific heat. The functions (1) are called the point spread functions (PSFs) of the $0^{\circ}$ and the $-90^{\circ}$ signal. Fig. 1 shows the profiles of these PSFs for silicon together with the $0 \%-90^{\circ}$ signal at a frequency of $88 \mathrm{~Hz}$. All curves are separately scaled here. We see that the $0^{\circ}$ signal is much stronger localized and actually diverges in source position, whereas the $-90^{\circ}$ signal only shows a weak maximum. Since the phase signal is given by $\Phi=\arctan \left(90^{\circ} / 0^{\circ}\right)$, the divergence of the $0^{\circ}$ signal at $r=0$ explains why the phase signal is always 0 in the position of a point source, independent on the magnitude of its power. On the other hand, the $0^{\circ} /-90^{\circ}$ signal very closely matches the $0^{\circ}$ signal, but in contrast to this it is not influenced by the emissivity contrast, which influences both the $0^{\circ}$ and the $-90^{\circ}$ signal in the same way. It can be said that the $0 \%-90^{\circ}$ signal uses the weakly localized $-90^{\circ}$ signal for the emissivity compensation of the highly localized $0^{\circ}$ signal. This works best in microscopic scale where the $-90^{\circ}$ signal is nearly constant.

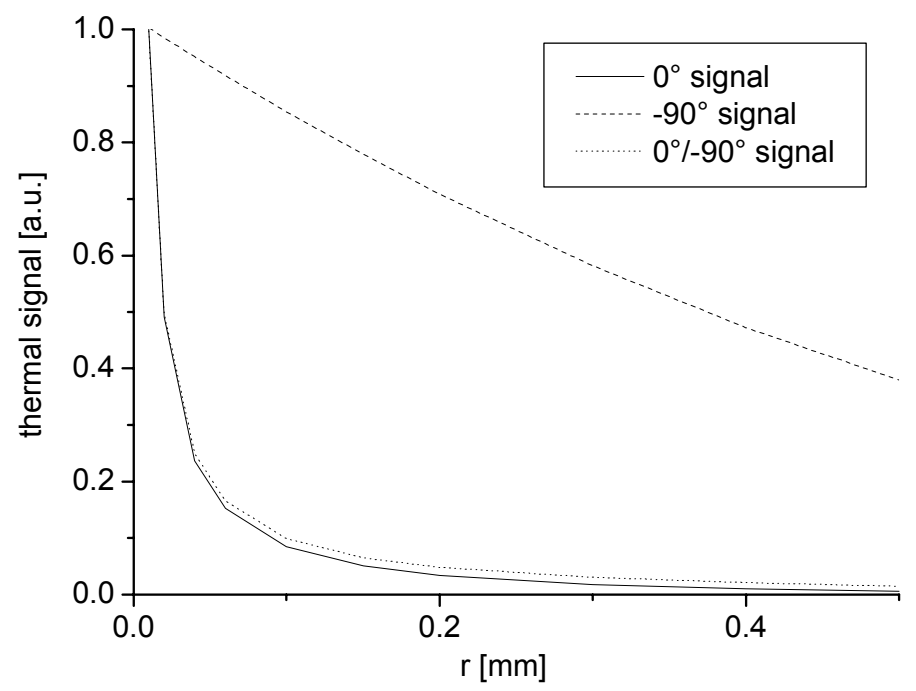

Fig. 1: Radial profiles of the $0^{\circ},-90^{\circ}$, and the $0 \%-90^{\circ}$ signal around a point source 


\section{http://dx.doi.org/10.21611/qirt.2004.002}

\section{Application to IC imaging}

The application of the proposed kind of image display is demonstrated in Fig. 2 , which shows lock-in thermograms taken with a microscope IR objective from an IC showing several local heat sources. Its supply voltage of $0.2 \mathrm{~V}$ was pulsed at $88 \mathrm{~Hz}$, during the bias pulses a current of $17 \mathrm{~mA}$ was flowing. Since a good signal-to-noise ratio was aspired, this measurement took about $12 \mathrm{hrs}$. As Figs. 2 (a) and (b) show, local heat sources are relatively well localized in the $0^{\circ}$ image, but the $-90^{\circ}$ image looks very much like a homogeneously heated area and basically displays the emissivity contrast. This contrast is avoided in the phase image (c), which, however, looks considerably blurred. Different heat sources with different magnitudes show a similar brightness in the phase image, as expected. The $0 \%-90^{\circ}$ image (d), on the other hand, is also free from emissivity contrast but shows a considerably better effective spatial resolution than the phase image (c). The reason is that the $0 \%-90^{\circ}$ signal basically represents the emissivity-corrected $0^{\circ}$ signal, which naturally has a better spatial resolution (see Fig. 1). Only the $0^{\circ} \%-90^{\circ}$ image clearly shows that besides the dominant heat source (strong arrow) there is a second point-like heat source in the upper right corner of one of the 6 dark metallized regions (weak arrow).
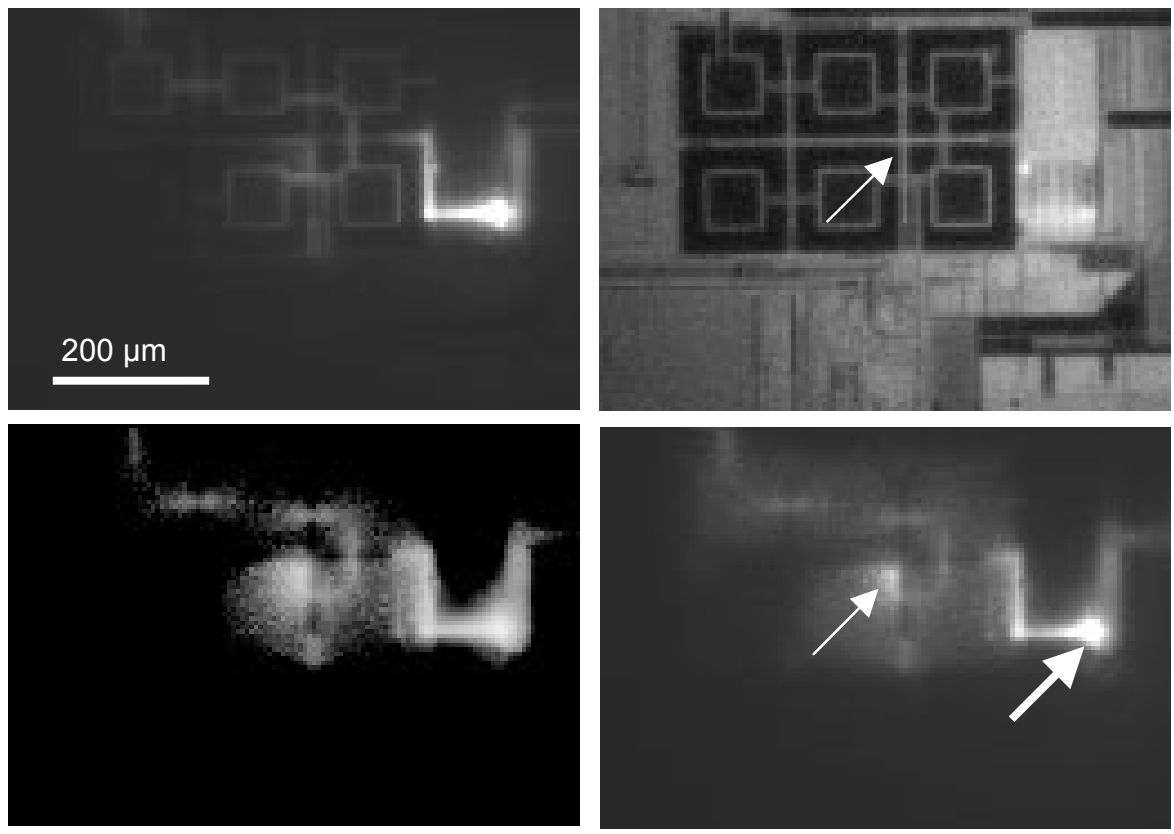

Fig. 2: $0^{\circ}$ image (a), $-90^{\circ}$ image (b), phase image (c), and $0 \%-90^{\circ}$ image (d) of an integrated circuit containing different local heat sources 


\section{Spatial deconvolution (inversion)}

Especially for microscopic lock-in thermography investigations the images are influenced by blurring, which is due to lateral heat diffusion from local heat sources into the sample area. As it has been explained in the introduction, only the $0^{\circ} /-90^{\circ}$ image is able to be deconvoluted, since only this image is free of emissivity contrast and behaves linearly with respect to different heat sources, which holds at least in microscopic approximation. Therefore this image (Fig. $2 \mathrm{~d}$ ) was deconvoluted using the $0^{\circ} \mathrm{PSF}$ with the result shown in Fig. 3. The standard technique for deconvoluting (inverting) lock-in thermograms is to use the in-phase and the out-of-phase signal in a complex FFT based procedure using Wiener filtering [7]. However, here we have only a scalar image to be deconvoluted. If the depth of the source in the sample is known (here it is at the surface) also scalar images can be deconvoluted, which can be done either by FFT-based techniques [7] or by iterative techniques working in real space. For performing the deconvolution of Fig. 2 (d) we have used an iterative procedure described in detail elsewhere [5]. It calculates a 2-dimensional power distribution $P(x, y)$ from a lock-in thermogram $T(x, y)$ using a certain point spread function (PSF). At the beginning as a first guess the power distribution $P_{1}(x, y)$ is assumed to be proportional to the measured $T$ signal distribution $T(x, y)$. Then from this power distribution a guessed $T$-distribution $T_{1}(x, y)$ is calculated by convoluting $\mathrm{P}_{1}(\mathrm{x}, \mathrm{y})$ with the chosen PSF. At the beginning of the procedure this temperature distribution will considerably deviate from the measured one $T(x, y)$. The difference between the convoluted and the measured $T$ distribution is used to correct the first guess $P$-distribution, leading to the second iteration $P_{2}(x, y)$. Now a "positivity constraint" can be implemented, setting all negative values of $P_{n}(x, y)$ to zero. This constraint considerably improves the convergence of the procedure and reduces the noise in regions without heat sources. If after a sufficient number of iterations the convoluted power comes close to the real power distribution, the convoluted distribution $T_{n}(x, y)$ approaches the measured distribution and the process converges. A comprehensive thermal deconvolution software "DECONV" enabling both FFTbased and iterative deconvolutions is available at one of the authors (O.B.). In the deconvoluted image shown in Fig. 3 the position of the secondary heat source is clearly defined to an accuracy of one pixel ( $5 \mu \mathrm{m}$, see arrow in Fig. 3).

\section{Conclusion}

A novel kind of representing lock-in thermograms is proposed, which is the display of the " $0 \%-90^{\circ}$ signal. This signal is not influenced by the local IR emissivity, but in contrast to the well-known phase image it behaves nearly linearly with respect 
to different local heat sources. This holds the better the smaller the investigated region is compared to the thermal diffusion length. Therefore this kind of representation is especially advantageous for microscopic lock-in thermography investigation of integrated circuits (ICs). It has been shown that $0^{\circ} /-90^{\circ}$ images even are able to become deconvoluted using the $0^{\circ}$ point spread function, leading to a considerable improvement of the effective spatial resolution.

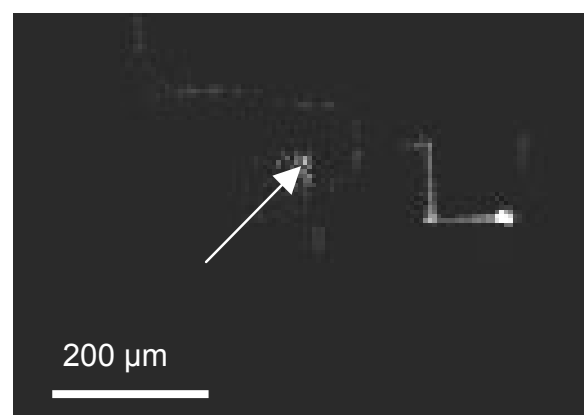

Fig. 3: Lateral power distribution numerically deconvoluted from Fig. 2 (d)

\section{REFERENCES}

[1] Farina, D.J. Making surface temperature measurements using liquid crystal thermography, Electronics Cooling 1, 10 (1995)

[2] Kolodner, P. and Tyson, J.A. Microscopic fluorescent imaging of surface temperature profiles with $0.01{ }^{\circ} \mathrm{C}$ resolution, Appl. Phys. Lett. 40, 782 (1982)

[3] www.quantumfocus.com

[4] Breitenstein, O. and Langenkamp, M. Lock-in Thermography - Basics and Use for Functional Diagnostics of Electronic Components, Springer, Heidelberg (2003)

[5] Breitenstein, O.; Konovalov, I. and Langenkamp, M. Highly-Sensitive Lock-in Thermography of Local Heat Sources using 2-Dimensional Spatial Deconvolution Proc. QIRT'2000 218-223 (2000)

[6] Carslaw, H.S. and Jaeger, J.C. Conduction of Heat in Solids, Clarendon, Oxford (1959)

[7] Seidel, U.; Haupt, K.; Walther, H.G.; Burt, J.A. and Munidasa, M. An Attempt Towards Quantitative Photothermal Microscopy, J. Appl. Phys. 78, 5828 (1993) 